\title{
Adolescents and HIV-related behaviour in Nigeria: does knowledge of HIV/AIDS promote protective sexual behaviour among sexually active adolescents?
}

\author{
Omoyeni, Sunday T.1, ${ }^{*}$, Akinyemi, Akanni I ${ }^{1,3}$ \& Fatusi, Adesegun 4 \\ Department of Demography and Social Statistics, Obafemi Awolowo University ${ }^{1}$ \\ International Organization for Migration (IOM), Abuja, Nigeria. ${ }^{2}$ \\ African Centre for Migration and Society (ACMS), University of Witwatersrand, \\ Johannesburg, South Africa ${ }^{3}$ \\ Department of Community Health \& Institute of Public Health, \\ Obafemi Awolowo University, Nigeria. ${ }^{4}$
}

\begin{abstract}
HIVIAIDS prevalence among Nigerian youth remains one of the highest in the world. Although studies confirmed a high knowledge of HIV among Nigerian youth, there is a lag in linking HIV/AIDS knowledge with protective sexual behaviour.

The study utilized data from the Nigeria 2007 National HIV/AIDS and Reproductive Health Survey (NARHS). Analysis was on 865 sexually active adolescents.

About $75 \%$ of adolescents had good knowledge of HIV/AIDS. Logistic regression models showed that HIV knowledge was significant predictor of non-use of condom $(O R=2.34 ; p=0.04 I)$ and accepting gifts for sex $(O R=2.02 p=0.043)$ among male, and predictor of multiple sexual partners $(O R=1.30 ; p=0.023)$ among females. Regardless of HIV knowledge, higher education, older age at first sex, higher wealth index and urban residency are significant predictors of engagement in non-protective sexual behaviour.
\end{abstract}

The study concludes that high knowledge of HIV/AIDS is not statistically significant in HIV protective attitudes among adolescents.

Keywords: HIV/AIDS, knowledge, sexual behaviour, adolescents, Nigeria

\section{Résumé}

La prévalence du VIH / sida chez les jeunes nigérian reste l'un des plus élevés au monde. Bien que des études ont confirmé bonne connaissance du VIH chez les jeunes nigérian, il ya un décalage de lier connaissance du $\mathrm{VIH} /$ sida et les comportements sexuels de protection.

Les données de l'étude utilisée de la nationale sur le VIH / sida de 2007 au Nigéria et Enquête sur la santé de la reproduction (NARHS). L'analyse a été sur 865 adolescents sexuellement actifs.

Environ $75 \%$ des adolescents avaient une bonne connaissance du VIH / SIDA. Des modèles de régression logistique ont montré que la connaissance du VIH était prédicteur important de non - utilisation de préservatif $(O R=2,34, p=0,04 I)$ et l'acceptation de cadeaux pour le sexe $(O R=2,02 p=0,043)$ chez les hommes, et un prédicteur de multiples partenaires sexuels $(O R=I, 30, p=0,023)$ chez les femmes. Peu de connaissances sur le VIH, l'enseignement supérieur, l'âge du premier rapport sexuel plus, un indice de richesse et de résidence en milieu urbain sont des prédicteurs significatifs de l'engagement dans le comportement sexuel non - protection .

L'étude conclut que bonne connaissance du VIH / sida n'est pas statistiquement significatif dans les attitudes de protection contre le VIH chez les adolescents.

Mots-clés: VIH / SIDA, les connaissances, le comportement sexuel, les adolescents, Nigeria

*. Corresponding author's e-mail: tunde.omoyeni@yahoo.com \& somoyeni@iom.int 


\section{Introduction}

HIV/AIDS pandemic remains a major public health problem in sub-Saharan African countries, particularly in Nigeria where more than 3 million people are currently living with the virus, with about $28 \mathrm{I}, 180$ new infections in 2010 (FMoH, 20l0). Intensive program intervention is believed to have yielded positive gains on curbing the spread of the disease a prevalence rate of $6 \%$ in year 2001 to $4.1 \%$ in 2010 . There are substantial variations in the burden of HIV/AIDS across regions, countries and population sub-groups. Studies have confirmed that youth are the most vulnerable to HIV/AIDs in sub-Sahara Africa (Fatusi \& Hindi, 20I0; Akinyemi \& Okpechi, 20II). However, the burden of HIV/AIDS among Nigerian youth still remains one of the highest in the world (FMoH, 20I0). The recent national estimates of (Sentinel, 20I2) HIV prevalence show that HIV prevalence rate for adolescents (I5-19 years) and youths (age 20-24 years) peaked at $3 \%$ and $4.6 \%$ respectively. These rates raise serious concerns about the protection of next generation.

Empirical researches from the developing countries converged on the findings that there is very high level of knowledge about HIV/AIDS, including prevention and risk (Cambell \& Mbivzo, 1994; MACRO-DHS, 2008; and Fatusi and Hindi, 20I0). Many of these studies also confirmed that HIV/AIDs is many sub-Saharan Africa is largely due to heterosexual activities, particularly those defined as high risk sex. So, the prevention of HIV is to a large extent zeroed on avoidance of risky sexual behavior and other high risk including sharing unsterilized needles and body piercing. Youth are critical group in terms of discourses around high risk sex (Akinyemi and Okpechi, 20I I).

Particularly in Nigeria, studies have documented that youth have very high knowledge of HIV/AIDs in terms of prevention and care (Odu \& Akanle, 2008; UNAIDS, 2008, COMPASS 2007, 2009). Juxtaposing the linkages between knowledge of HIV/AIDS and its implications for responsive sexual-related behaviour among adolescents in Nigeria remain a major gap. Majority of studies have focused on the socio-demographic and psychosocial factors influencing onset of sexual and risky sexual behaviour among adolescents (Santelli et al. 2004; Fatusi \& Blum, 2008). To a large extent, few studies that made attempts in explaining the pathway of knowledge of HIV/AIDS and sexual behaviour have been limited to in-school adolescents with a very limited coverage (Okunta \& Oseji, 2006), overlooking the majority of out of school adolescents.

Consistent use of condom during casual sex and having one faithful sexual partner have been found to be an effective way of preventing the transmission of HIV/AIDS among men and women, and particularly among youth at the onset of pre-marital sex (Abma and Mcgill, 2007). Additionally, late sexual debut among adolescents is linked with reduced lifetime prevalence of sexual partners, thereby reducing the risk of exposure to sexually transmitted diseases (STDs), including HIV/AIDS and unwanted pregnancy. In Nigeria as in many other developing countries, socio-cultural norms preventing adolescents from accessing preventive measures and safe sex practices are still prevalent. Young people are therefore within a web of translating abstract knowledge of HIV/AIDs prevention into to actual behavior considered as safe.

The crux of this current thought therefore, is to appraise the implications of HIV/AIDS knowledge for safe sexual practices among sexually active adolescents in Nigeria. The proposition here is that knowledge of HIV/AIDS is to a large extent expected to influence protective sexual behaviour in a pathway that will reduce HIV-risky behavior, particularly as it relates to sex. The central question for the analysis is to find out if the knowledge of HIV/AIDS in terms of modes of transmitting and prevention among adolescents translate into safer sexual behaviour or not? Also, are there significant mediating variables in young people sexual behaviour independent of their level of HIV knowledge? Specifically, the paper is aimed at an attempt to provide some lucid analysis and explanations to this argument.

\section{Data and methods}

The study utilized data from the Nigeria 2007 National HIV/AIDS and Reproductive Health Survey (NARHS). NARHS is a nationally representative survey females aged 15-49 years and males aged 15-64 years of the reproductive age. The study population was randomly selected using three levels, multistage method with the aim of selecting eligible respondents in each of the 36 states in Nigeria with equal probability. The selection of respondents began with stratification of locations into rural and urban residences, followed by random selection of enumeration areas, and subsequently individuals. A sample of 11,251 individuals $(53.5 \%$ for males and $46.5 \%$ for females) was selected at the household level in NARHS 2007. Out of 2,470 adolescent (age 15-19 years) population sampled, the study utilizes data on 865 never married sexually active adolescents who have had sexual intercourse in the last 12 months preceding the survey period.

The main independent variable for the study is adolescents' knowledge of HIV/AIDS and this was assessed from information on seven modes of trans- 
mission and prevention of HIV/AIDS ${ }^{* * *}$ as reported by the adolescents. Factor analysis technique was used to categorize the level of knowledge into high, medium and low level. The reliability coefficient of item loadings is displayed in Table I. The other independent variables consisted of educational level, place of residence, wealth index, religion and region. Also included in the analysis are some sexual behaviour variables such as age at first sex, sex partner age grade, discussion of HIV/AIDS. The outcome variable for the study was HIV non-protective sexual behaviour measured by the use of condom during last sexual intercourse in the last 12 months, having more than one sexual partner and exchange of gifts for sex with a strange person. The analysis was further disaggregated into male and female sex categories. The method of analysis was done at three levels of univariate, bivariate and multivariate analyses.

Data were analysed using STATA (version II.0). Data analysis was carried in three levels and further disaggregated into sex. First, the univariate analysis was undertaken to profile the proportion of sexually active adolescents with respect to level of HIV knowledge, age at first sex, sex partner age grade and other selected background characteristics. Chisquare was used to assess association between level of HIV knowledge and non-protective sexual behavior. The analysis also involved the determination of selected background characteristics that showed significant association with condom use, accepting gifts for sex and having more than one sexual partner. The percentage of adolescents with non-protective sexual behavior across selected variables and corresponding chi-square values was tabulated. A significance value of selected independent and background variables was established at $p$-value $<0.05$.

Multivariate analysis was performed using binary logistic regression analysis to predict risk factors of engaging in HIV non-protective sexual behavior. Two models for each of the HIV non-protective sexual behavior variables for males and females separately were simulated: the first included only the main independent variable (level of HIV knowledge), while the second model included level of HIV knowledge and other selected background variables. Only the odd ratios of the variables simulated were presented in Tables four and five.

\section{Results}

Background characteristics of the respondents

Table I Background characteristics and sexual behavior of Adolescents by Sex

\begin{tabular}{llll}
\hline Variables & Male (\%) & Female (\%) & Both (\%) \\
\hline Educational level & & & 10.2 \\
Primary or lower & 10.0 & 11.0 & 89.8 \\
Secondary or higher & 90.0 & 89.0 & 40.1 \\
Place of residence & & & 59.9 \\
Urban & 39.6 & 43.2 & \\
Rural & 60.4 & 56.8 & 40.7 \\
Wealth Status & & & 28.4 \\
Low & 37.5 & 44.8 & 30.9 \\
Average & 29.2 & 26.8 & \\
High & 33.3 & 28.4 & 40.7 \\
Religion & & & 59.3 \\
Non-protestant & 42.7 & 37.4 & \\
Protestant & 57.3 & 62.6 & 27.6 \\
Region & & & 72.4 \\
\hline North & 30.5 & 20.2 & 7.3 \\
South & 69.5 & 56.8 & 18.0 \\
Level of knowledge & & 19.7 & \\
Low & 6.1 & 16.3 & \\
Medium & 16.2 & & \\
& & & \\
\hline
\end{tabular}

** HIV can be transmitted through unprotected sexual intercourse; HIV transmission can be prevented by delaying onset of sexual intercourse; HIV transmission can be prevented by avoiding sex with commercial sex workers; HIV transmission can be prevented by using condom during sexual intercourse; HIV transmission can be prevented by reducing number of sexual partners; HIV transmission can be prevented by abstaining from sex; HIV transmission can be prevented by avoiding sex with multiple partners. 
High

Away from home in the last 12 months

Yes

No

Sexual behavior variables

Age at first sex

15 or lower

16-19

Median age at first sex

Sex partner age grade

Younger

About the same age

Older than me

Ever discussed HIV/AIDS with sexual partners*

Ever discussed HIV/AIDS with neighbor*

Ever discussed HIV/AIDS with parents*

*Only the percentage for "yes" is tabulated

Almost 9 out of every 10 respondents have at least secondary education $(90 \%$ for males and $89 \%$ for females). About $60 \%$ reside in the rural communities with about $41 \%$ in the lowest wealth tertile. Evidence of low wealth status is found to be higher among male adolescents (38\%) than female counterparts (45\%). About $72 \%$ were sampled from the Southern part of Nigeria while only about $28 \%$ were from Northern states. The median age at first was estimated at 17 years $33 \%$ had debuted sex by age
33.0

67.0

$4 I .5$

39.3

40.4

58.5

60.7

59.6

17 years

33.8

33.3

66.2

66.7

17 years

$\begin{array}{lll}70.9 & 4.6 & 47.3\end{array}$

$\begin{array}{lll}24.4 & 17.8 & 22.0\end{array}$

$\begin{array}{lll}4.7 & 77.6 & 30.7\end{array}$

$\begin{array}{lll}48.4 & 43.3 & 46.7\end{array}$

$\begin{array}{lll}33.1 & 34.5 & 33.7\end{array}$

$17.9 \quad 31.1 \quad 22.2$

\section{Table 2}

Measurement scales, Internal Consistency and Item Loadings

\begin{tabular}{ll}
\hline Scale Name & Item content \\
\hline $\begin{array}{l}\text { HIV knowledge (Reliability coefficient) }= \\
.764\end{array}$ & HIV can be transmitted through unprotected sexual intercourse .790 \\
\hline & HIV transmission can be prevented by delaying onset of sexual .640 \\
& intercourse \\
& HIV transmission can be prevented by avoiding sex with commercial .566 \\
& sex workers \\
& HIV transmission can be prevented by using condom during sexual .772 \\
& intercourse \\
& HIV transmission can be prevented by reducing number of sexual .425 \\
& partners. \\
& HIV transmission can be prevented by abstaining from sex \\
& HIV transmission can be prevented by avoiding sex with multiple \\
& partners \\
&
\end{tabular}

Table 2 presents the composite measure of index of HIV knowledge. Seven variables related to knowledge of transmission and prevention of HIV/AIDs was utilized. The reliability coefficient using Cronbach Alpha was 0.764 and each of the item loading was as presented in Table 2. Using factor analysis, three levels of knowledge of HIV/AIDs were com-
15 years or less. More than $70 \%$ of young men had first sexual experience with younger girls relatively younger to their ages while only $5 \%$ of girls had first sex with boys relatively younger than their ages. The results further showed that communication about HIV AIDs is usually discussed with sexual partners (47\%), neighbors (34\%), and parents (22\%) Almost half of the respondents $(48.4 \%$ for males versus $43.3 \%$ for females) ever discussed HIV/AIDS with their partners. 
Association between selected factors and HIV non-protective sexual behavior: males (Table 3)

Table 3 Association between selected factors and HIV non-protective sexual behavior: males

\begin{tabular}{|c|c|c|c|c|c|c|}
\hline \multirow[t]{2}{*}{ Variable } & \multicolumn{2}{|c|}{ use of condom } & \multicolumn{2}{|c|}{ Accept gift for sex } & \multicolumn{2}{|c|}{ More than one partner } \\
\hline & $\%$ & Chi-square & $\%$ & Chi-square & $\%$ & Chi-square \\
\hline \multicolumn{7}{|l|}{ Level of knowledge } \\
\hline Low & - & & 5.7 & & 40.0 & \\
\hline Medium & 3.3 & 0.222 & 19.0 & $0.049 *$ & 46.7 & 0.670 \\
\hline High & 1.2 & & 10.9 & & 41.9 & \\
\hline \multicolumn{7}{|l|}{ Educational level } \\
\hline Primary or lower & 2.0 & $0.032^{*}$ & 18.9 & $0.010 *$ & 49.1 & 0.312 \\
\hline Secondary or higher & 1.4 & & 11.2 & & 41.9 & \\
\hline \multicolumn{7}{|l|}{ Place of residence } \\
\hline Urban & 1.0 & $0.043^{*}$ & 14.1 & 0.195 & 45.5 & 0.258 \\
\hline Rural & 2.0 & & 10.5 & & 40.6 & \\
\hline \multicolumn{7}{|l|}{ Wealth Status } \\
\hline Low & 2.4 & $0.003^{*}$ & 13.5 & 0.447 & 41.8 & 0.480 \\
\hline Average & 0.6 & & 9.3 & & 46.3 & \\
\hline High & I.I & & 12.4 & & 40.0 & \\
\hline \multicolumn{7}{|l|}{ Religion } \\
\hline Non-protestant & 1.2 & 0.764 & II.4 & 0.754 & 40.9 & 0.512 \\
\hline Protestant & 1.6 & & 12.3 & & 43.7 & \\
\hline \multicolumn{7}{|l|}{ Region } \\
\hline North & 2.0 & 0.663 & 8.9 & 0.146 & 42.6 & 0.980 \\
\hline South & 1.3 & & 13.2 & & 42.5 & \\
\hline \multicolumn{7}{|c|}{ Ever been away from home } \\
\hline Yes & 2.2 & 0.225 & 12.3 & 0.701 & 43.0 & 0.829 \\
\hline No & 1.0 & & 11.2 & & 42.1 & \\
\hline \multicolumn{7}{|l|}{ Age at first sex } \\
\hline 15 or lower & 1.8 & 0.786 & 13.5 & $0.034 *$ & 48.5 & $0.004 *$ \\
\hline $16-19$ & 2.0 & & 11.5 & & 40.1 & \\
\hline Summary of result & 1.4 & & 15.5 & & 42.5 & \\
\hline
\end{tabular}

*Association is significant at $\mathrm{p}<0.05$

Out of eight selected explanatory variables included in the bivariate analysis, level of HIV/AIDS knowledge, education, place of residence and wealth status were significantly associated $(p<0.05)$ with condom use during sexual intercourse in the last 12 months. About $1.2 \%$ of adolescents with high knowledge of HIV reported the use of condom last sexual intercourse. Surprisingly, the magnitude of adolescents who reported the use of condom during last sexual intercourse decrease with the level of education. Only $2 \%$ and $1.4 \%$ of male adolescents with primary or lower education respectively used condom during last 12 months. Association between level of education and condom use was significant $(p=0.032<0.05)$. Also, about $2 \%$ of male adolescents from rural areas reported the used of condom compare to $1 \%$ of male adolescents from urban locations. With respect to wealth status, high con- dom during last sexual intercourse was found among those in low wealth status $(2.4 \%)$ relative to those in medium $(0.6 \%)$ and high ( $1.1 \%)$ wealth categories.

Predictors of acceptance of gifts for sex, showed that level of HIV knowledge, educational level and age at first had significant associations $(p<0.05)$. As evidence from table 3, about $6 \%, 19 \%$ and $11 \%$ of male adolescents with low, medium and high level of HIV knowledge respectively reported ever received gifts for sex. Higher proportion of male adolescents with primary or lower education (19\%) than those with secondary school or higher (II\%) reported acceptance of gifts for sex. Higher proportion of those reported accepting of gifts for sex was found among male adolescents who initiated sex at age 15 or lower (14\%) compared to those at 16-19 years (I2\%). The association is between age at first sex and accepting gifts for sex was significant at $p<0.05$. 
Higher proportion of male adolescents with medium level of HIV knowledge (47\%) compared to those with low (40\%) and high (42\%) level of HIV knowledge reported having more than one sexual partner. Male adolescents with primary or lower education (49\%) are more likely to report having more than one sexual partner compared to those with secondary or higher education (42\%). No statistical difference was observed. With respect to place of residence, about $45 \%$ of male adolescents who reside in urban areas reported having more than one sexual partner compared to $41 \%$ of those from rural areas. High proportion of male adolescents in average wealth group (46\%) relative to those in low (42\%) and high (40\%) wealth group reported having more than one sexual partner. Out of the eight variables included in the analysis, only age at first showed significant association with risk factor of having more one sexual partner. Overall, $1.5 \%, 11.9 \%$ and $42.5 \%$ of sexually active male adolescents reported the use of condom, accepting gifts for sex and having more than one sexual partner respectively.

\section{Association between selected factors and HIV non-protective sexual behavior: females (Table 4)}

Table 4 Association between selected factors and HIV non-protective sexual behavior: females

\begin{tabular}{|c|c|c|c|c|c|c|}
\hline \multirow[t]{2}{*}{ Variable } & \multicolumn{2}{|c|}{ use of condom } & \multicolumn{2}{|c|}{ Accept gift for sex } & \multicolumn{2}{|c|}{ More than one partner } \\
\hline & $\%$ & Chi-square & $\%$ & Chi-square & $\%$ & Chi-square \\
\hline \multicolumn{7}{|l|}{ Level of knowledge } \\
\hline Low & 3.0 & & 12.1 & & 15.1 & \\
\hline Medium & 5.0 & $0.037^{*}$ & 20.0 & 0.552 & 16.7 & 0.961 \\
\hline High & 0.5 & & 15.2 & & 15.2 & \\
\hline \multicolumn{7}{|l|}{ Educational level } \\
\hline Primary or lower & 5.9 & $0.036 *$ & 23.5 & $0.019 *$ & 20.1 & 0.383 \\
\hline Secondary or higher & 1.1 & & 14.7 & & 14.9 & \\
\hline \multicolumn{7}{|l|}{ Place of residence } \\
\hline Urban & 2.3 & 0.445 & 10.5 & $0.024^{*}$ & 9.7 & $0.014 *$ \\
\hline Rural & I.I & & 20.0 & & 19.9 & \\
\hline \multicolumn{7}{|l|}{ Wealth Status } \\
\hline Low & 1.4 & \multirow{3}{*}{0.168} & 18.7 & \multirow{3}{*}{0.424} & 20.1 & \multirow{3}{*}{$0.012 *$} \\
\hline Average & 3.6 & & 14.5 & & 14.5 & \\
\hline High & - & & 12.5 & & 9.1 & \\
\hline \multicolumn{7}{|l|}{ Religion } \\
\hline Non-protestant & 1.7 & \multirow[t]{2}{*}{0.904} & 12.9 & 0.283 & 16.4 & \multirow[t]{2}{*}{0.736} \\
\hline Protestant & 1.6 & & 17.5 & & 15.0 & \\
\hline \multicolumn{7}{|l|}{ Region } \\
\hline North & 3.2 & \multirow[t]{2}{*}{0.270} & 15.9 & 0.987 & 16.0 & \multirow[t]{2}{*}{0.924} \\
\hline South & 1.2 & & 15.8 & & 15.0 & \\
\hline \multicolumn{7}{|c|}{ Ever been away from home } \\
\hline Yes & 1.7 & \multirow[t]{2}{*}{0.973} & 15.1 & 0.691 & 12.6 & \multirow[t]{2}{*}{0.215} \\
\hline No & 1.6 & & 16.9 & & 17.9 & \\
\hline \multicolumn{7}{|l|}{ Age at first sex } \\
\hline 15 or lower & 1.0 & \multirow[t]{3}{*}{$0.024 *$} & 21.8 & $0.040 *$ & 18.8 & \multirow[t]{3}{*}{0.353} \\
\hline $16-19$ & 2.0 & & 12.6 & & 14.7 & \\
\hline Summary of result & 1.6 & & 15.5 & & 16.0 & \\
\hline
\end{tabular}

*Association is significant at $p<0.05$

This section deals with explanation of associations between selected factors and female adolescent HIV non-protective behavior. The analysis was done by cross tabulating selected independent variables with the use of condom during last sex, accepting gift for sex and having more than one sexual partner. The result showed evidence of significant associations between female adolescents' level of HIV knowledge and use of condom. The degree of condom use is very low among the respondents. About $1 \%$ of female adolescents with high knowledge of HIV reported having used condom during last sex in the 
last 12 months. Among this group, female adolescents with medium level of HIV knowledge reported higher use of condom (5\%). Educational is another factor that showed significant association with condom use $(p=0.036<0.05))$. Surprisingly, higher proportion of female adolescents with primary or lower education $(6 \%)$ than those with secondary or higher education ( $1 \%$ ) reported having used condom. With respect to wealth status, condom use is lower among those in low wealth group (I.4\%) than those who in medium wealth group (4\%), although the association is not statistically significant. Similarly, adolescents from Northern region (3\%) reported higher use of condom than those from the Southern region (1.2\%). Female adolescents' age at first sex showed significant association with the use of condom $(p=0.024)$. Condom use is found to be higher among female adolescents who initiated sex at 16-19 (2.0\%) compared to those who reported sex debut at 15 or lower (1.0\%).

Female adolescents' level of HIV knowledge showed no significant relationship with accepting gifts for sex. However, the proportion of female adolescents who reported ever received gifts with respect to level of HIV knowledge is substantial. About $12 \%, 20 \%$ and $15 \%$ of adolescents with low, medium and high knowledge of HIV respectively reported accepting gifts for sex. The proportion of female adolescents with primary or lower education who reported ever received gifts for sex $(24.0 \%)$ is higher than those in secondary or higher education who reported action attitude (I5\%). Association between level of education and accepting gifts for sex is significant $(p=0.019)$. More rural female adolescents $(20.0 \%)$ than those in urban settings (11\%) reported accepting gifts for $\operatorname{sex}(p=0.024)$. Similarly, female adolescents who initiated sex at age 15 or lower $(22 \%)$ are more likely to report accepting gifts for sex than those who initiated sex at older ages (13\%) and the association between age at first sex and risk of accepting gifts for sex is significant at $\mathrm{p}<0.05$.

Predictors of the risk of having more than one sexual partner showed that place of residence and wealth status had significant associations with multiple sexual partners. The result showed that about $20 \%$ of female adolescents from rural areas compared to $10 \%$ of those from urban locations reported having more than one sexual partner. Also, the proportion of adolescents who reported having more than one sexual partners decreases with increasing wealth status $(20.0 \%, 15.0 \%$ and $9.1 \%$ for those in low, medium and high wealth group respectively). In summary, the proportion of female adolescents who reported condom use, accepting gifts for sex and having more than one sexual partner is $1.6 \%, 15.5 \%$ and $16.0 \%$ respectively.

\section{Result of multivariate analysis}

This section presents the multivariate analysis of variables. Binary logistic regression analysis was used to predict the effects of independent variables on the risk of engaging in HIV non-protective sexual behavior. The major independent variables for the study are the level of HIV knowledge and some selected background characteristics. The dependent variable - HIV non-protective behavior - was measured by use of condom, accepting gifts for sex and having more than one sexual partner. Each of the levels of the dependent variables in the multivariate analysis was coded " 0 " if the respondent did not report risk factor of engaging in non-protective sexual behaviour and " $\mathrm{l}$ " if the respondent reported risk factor of engaging in non-protective sexual behaviour.

\section{Logistic regression models predicting odds of engaging in HIV non- protective sexual behavior: males (Table 5)}

Two models each of binary logistic regression were simulated. In Model I, surprisingly, the odds of reporting non-use of condom increased significantly by $28 \%$ and $134 \%$ among male adolescent with medium and high knowledge of HIV respectively compared to those with low knowledge of HIV. In model 2, after controlling for the effects of some background characteristics, the odds of not using condom increased significantly by $63 \%$ and $210 \%$ among those with medium and high level of HIV knowledge respectively relative to those with low level of knowledge. With respect to age at first sex, male adolescents who initiated sex at age 16-19 years are less likely $(0.33)$ report to non-use of condom compare to those in the reference category (age less than 16 years). Distributions across place residence show that male rural adolescents compared to urban counterparts have higher odds of reporting non-use of condom. The odds increased by $37 \%$ among rural adolescents. Protestant adolescents are significantly more likely (I.27) to report non-use of condom compared to the non-protestant. 


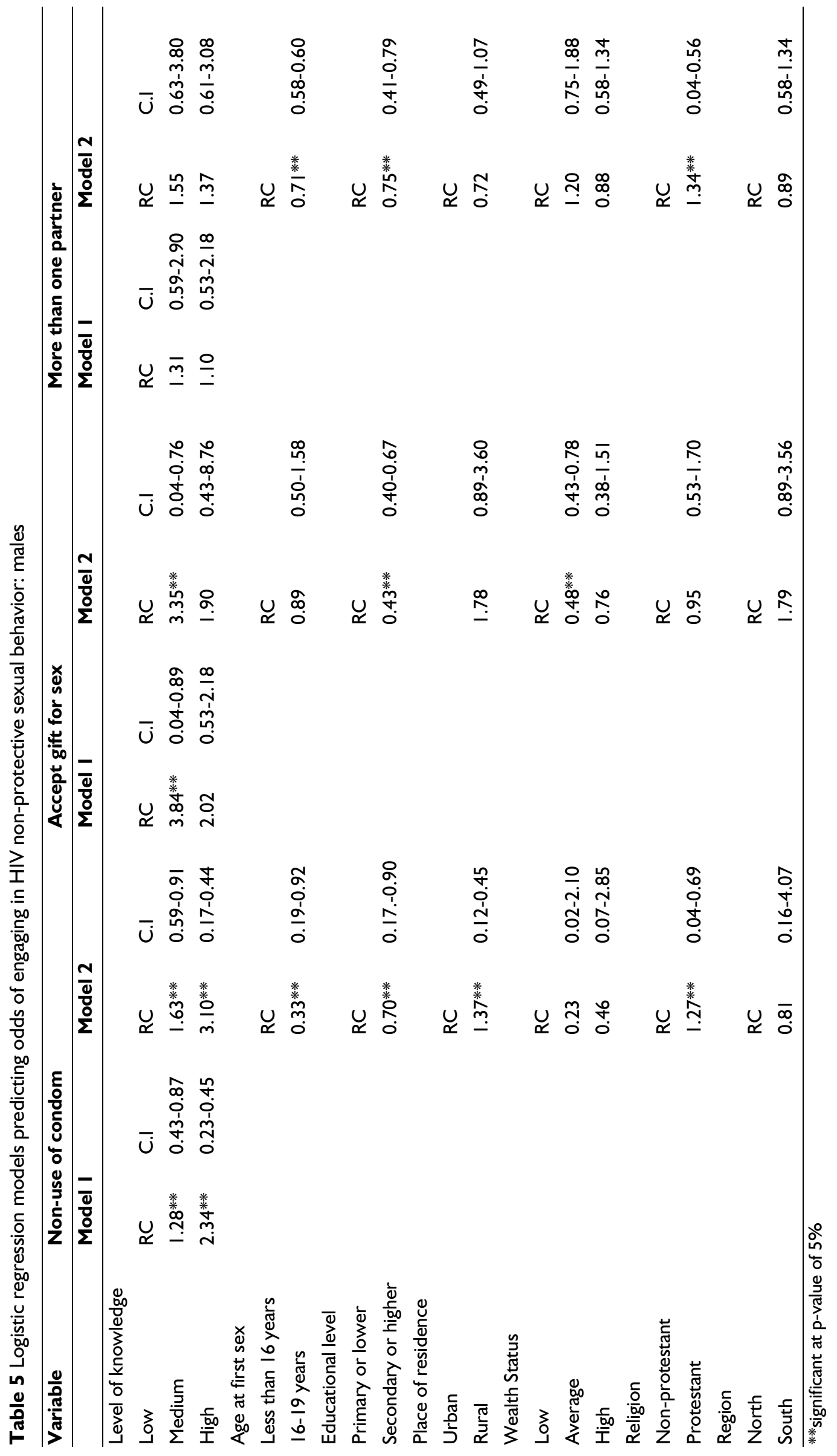




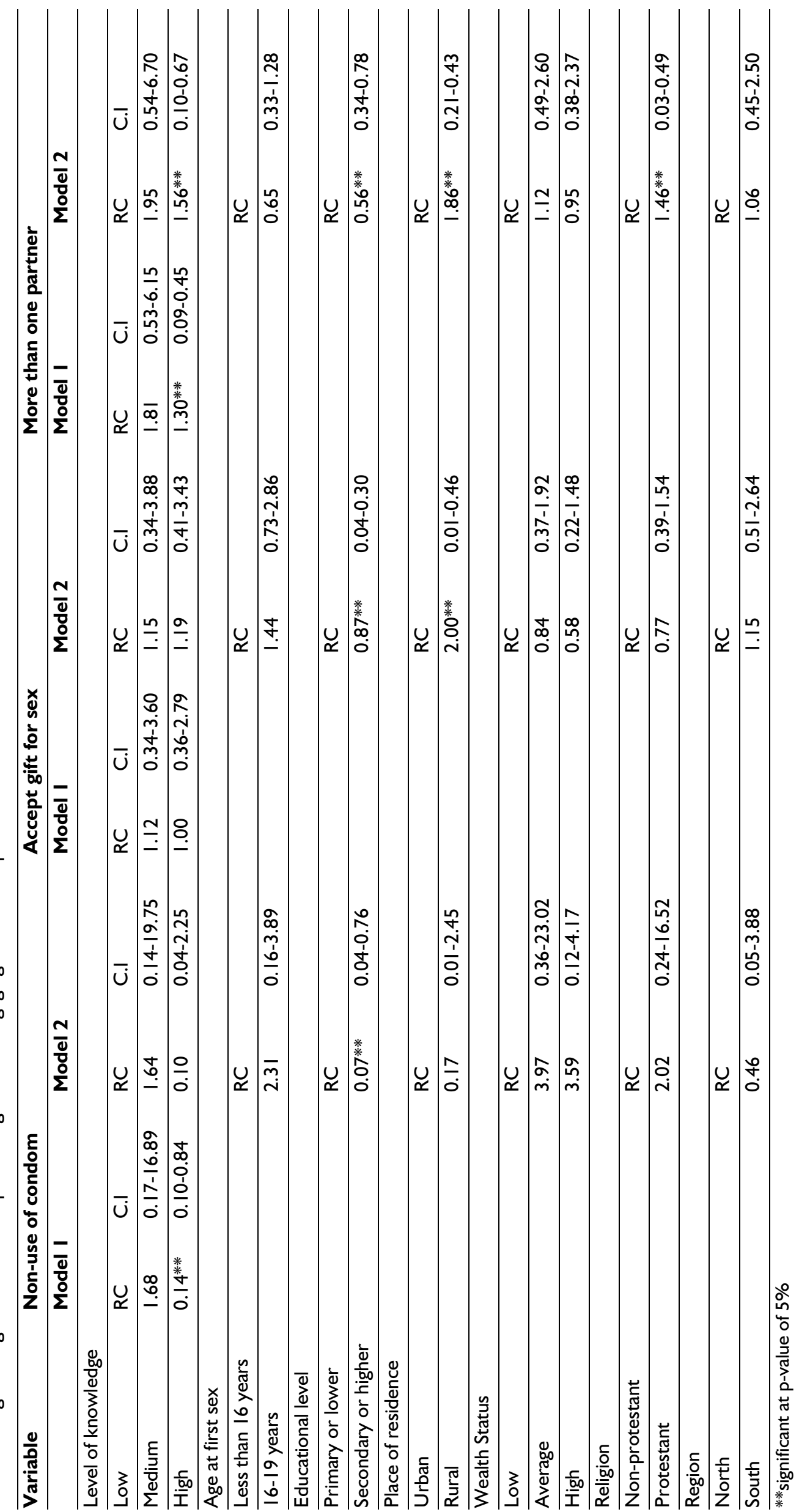


With respect to accepting gifts for sex, in model one, male adolescents with medium level of knowledge of HIV are 3.84 times as likely as those with low knowledge to report accepting gifts for sexual intercourse. In the second model, the odds of accepting gifts for sex among those with medium level of HIV knowledge increased significantly by $235 \%$ compared with those with low level of knowledge. Similarly, male adolescents with secondary or higher education are 0.43 times as likely as those with primary or lower education to report accepting gifts for sex. The odds of reporting accepting gifts for sex decreased significantly by $52 \%$ among those in medium wealth group compared to those in the reference group.

Male adolescents who initiated sex at age 16-19 years are significantly less likely $(0.7 \mathrm{I})$ to report more than one sexual partner compared to those who initiated sex at younger ages. Male protestant Christians are 1.34 times as likely as their non-protestant counterparts to report more than one sexual partner.

\section{Logistic regression models predicting odds of engaging in HIV non- protective sexual behavior: females (Table 6)}

The odds of not using condom decreased significantly by $86 \%$ among female adolescents with high level of knowledge of HIV compared to those with low level of knowledge. In the second model, only educational level significantly predicted the odds of non-use of condom. The odds indicates that female adolescents with secondary or higher education are 0.07 times as likely as those with primary or lower education to report non-use of condom. Out of 7 predictor variables, only educational level and place of residence significantly explained than the risk of accepting gifts for sex. Female rural adolescents are twice as likely as urban counterparts to report accepting gifts for sex.

Level of HIV knowledge is significant factor predicting the likelihood of having more than one sexual partner among female adolescents. Stunningly, female adolescents with high level of HIV knowledge are 1.56 times as likely as those in reference category to report having more than one sexual partner. Similarly, relative to urban female adolescents, those from rural areas reported higher odds of having more one sexual partner. The odds of reporting more than one sexual partner increased significantly by $46 \%$ among protestant compared to non-protestant counterparts.

\section{Discussion and conclusion}

The study had focused on the relationships between knowledge of HIV and HIV-related sexual behaviour using a nationally representative sample of Nigerian adolescents. Knowledge of HIV was measured from the information on seven indicative questions of how to prevent transmission and promote prevention measures of HIV. Evidence from the study shows that majority of adolescents have high knowledge of modes of contracting and transmitting HIV/AIDS. This finding is consistent with other studies that have documented high knowledge of HIV/AIDS among Nigerian adolescents (Omeonu \& Kollie, 20I0). High level of HIV knowledge in Nigeria as documented in this study and other past studies shows efficiency in the health education programme on HIV in Nigeria. When disaggregated by sex, the study shows huge knowledge gap between males and females, with higher proportion of males than females reported high knowledge of HIV. The present finding underscores gender imbalance in the level of HIV knowledge among adolescents. This finding is not consistent with Cheng, Eke-Huber, Eaddy and Collins (2005) who reported in their study that female students had a higher overall knowledge on HIV than males; however, Lowndes et al. 2008 in a different study in western Africa, reported that males have higher level of HIV knowledge than females but none of these studies have made recourse to factors accounting for the differentials.

Overall, the proportion of males and females that reported the use of condom during the last sexual intercourse in the last 12 months was estimated at $1.5 \%$ and $1.6 \%$ respectively. Evidence of low use of condom among sexually active adolescents as found in this study agrees with other previous studies (Fatusi \& Blum, 2008; Adebiyi \& Asuzu, 2009; Oyediran et al. 20I I). Surprisingly, the study found evidence of low condom use among male and female adolescents with high knowledge of HIV/AIDS and different level of background factors considered in this study. This finding reiterates the need for extensive and efficient behavioural change communication programmes with emphasis on education on sexual abstinence and safe-sexual behaviour as being currently undertaken by some Non-Governmental Organizations (NGOs), Civil Society Organizations (CSOs) and faith-based organizations in Nigeria.

The result shows gender differences in the factors associated with the use of condom. Educational level, place of residence and wealth status show significant association with use of condom during last sexual intercourse for males while level of HIV knowledge, educational level and age at first sex are significantly associated with condom use for females. 
The finding emphasizes strengthening programmes on sex and sexual related education among adolescents. The significant association of age at first sex and condom use for females support already documented evidence that female adolescents with early age at sexual initiation lack the will and power to negotiate safe sex with their partners (Onayade et al. 2008), hence, gender focused programmes, particularly on female adolescents to safe sex practices should be vigorously pursued.

Consistent with previous studies, higher proportion of female adolescents (42\%) than males (16\%) reported having more than one sexual partner. This finding questions some assertions that females consistently under report and males over report their sexual activities. In terms of the relationship between adolescents background characteristics and likelihood of having more than one sexual partner, age at first sex shows significant association with having more than sexual partner $(p=0.004<0.05)$ while place of residence and wealth status show significant association for female adolescents. The significant association of age at first sex with number of sexual partner further exemplifies assertion that the number of sexual partner that a person will have in her lifetime is hinged upon the age at which sexual act is initiated. The earlier the age at first sex, the more the number of sexual partner a person would have. This assertion was documented in the work of (Sanjose et al. 2008). Similarly, the established correlate of number of sexual partner with place of residence and wealth status, particularly for female adolescent further support previous findings of higher risk sexual behaviour among adolescents in rural areas (Voeten et al. 2004) and those from low wealth status background as a result of lack socioeconomic will to negotiate safe sex with their partners (Booysen and Summerton, 2002).

In the multivariate analysis, level of HIV knowledge significantly predicted the likelihood of non-use of condom and accepting gift for sex in the male model but only predicted the odds of having more than one sexual partner in the female model. As evidence in the study, high level of HIV knowledge does not constraint the adolescents from engaging in risky sexual behaviour such as non-use of condom, accepting gifts for sex from unknown partner and having more than one sexual partner. In other words, the prevalence of risky sexual behaviour is found among adolescents with higher knowledge of HIV/AIDS. This finding is consistent with the work of Letamo Gobopamang (20II) among young people in Bostwana, where he found that correct knowledge of HIV does not necessarily translate into protective sexual behaviour and Booysen and Summerton (2002) study on South African women aged 15-49 years. Regardless of adolescents' level of HIV knowledge, those with higher education, older age at first sex, higher wealth index and from urban location are less likely to engage in HIV non-protective sexual behaviour compared to adolescents in other categories. This finding suggests that the provision of HIVrelated information alone is not necessarily enough for adolescents to adopt safer sexual practices or to be better able to protect themselves from HIV. However, access to economic resources appears to improve the ability of adolescents to negotiate sexual relationships in a way that allows them to put their HIV-related knowledge into practice and consequently avoid infection.

Despite widespread knowledge of HIV/AIDS among adolescents as evident in this study, high risk sexual behaviour (non-use of condom, accepting gifts for sex and having more than sexual partner) is still prevalent. Majority of the adolescents also show evidence of lack of communication in discussing their sexual lives with their parents. Therefore, the study recommends that beyond interventions for HIV/ AIDS awareness programs and advocacies, targeted programmes in schools and out of schools should focus more on promoting safe sex practices among adolescents, particularly among those who lack social and economic will to negotiate safe sex behaviour with their partners. Attention should also be drawn to bridging the communication gaps between adolescents and their parents, through child-parent behavioural change communication programmes on sexuality education.

\section{Acknowledgment}

Authors acknowledged the Federal Ministry of Health, Nigeria for granting us permission to use the 2007 National HIV/AIDS and Reproductive Health Survey (NARHS) dataset. We also appreciated the William and Flora Hewlett Foundation, the Bill and Melinda Gates Foundation and the Population Association of America (PAA) Fund for providing financial support to present this paper at the 20I2 PAA meeting, San Francisco, California.

\section{References}

Abma, J \& McGill B (2007): "Teenagers' use of contraceptives at first intercourse: Long term trends in use, correlates and predictors for males and females". Paper presented at the Population Association of America meeting, New York, USA. Adebiyi AO and Asuzu MC (2009): "Condom use amongst out of school youths in a local government area in Nigeria". African Health Science. 2009 June; 9(2): 92-97.

Akinyemi Al and Okpechi FU (20I I): "Age, class, sex 
and the danger between: socio-economic inequalities and sexual \& reproductive vulnerabilities of young women in Africa". In: Sexuality in Africa Magazine and Monographs, pp 9-II

Booysten F \& Summerton J (2002): Poverty, risky sexual behaviour, and vulnerability to HIV infection: evidence from South Africa. Journal of Health Population and Nutrition. Volume: 20 Source Issue: 4

Campell B \& Mbivzo (1994): "Sexual behaviour and HIV knowledge among adolescent boys in Zimbabwe". Central African Journal of Medicine, 1994 Sep; 40(9):245-50

Chang, CL, Eke-Huber E, Eaddy S, \& Collins, JR (2005): "Nigerian college students: HIV knowledge, perceived susceptibility for HIV and sexual behaviours". College Student Journal, 39

De Sanjose S, et al (2008): "Age at sexual initiation and number of sexual partners in the female Spanish population", Eur. J. Obstet. Gynecol, doi:10.1016/j.ejogrb.2008.04.005

Fatusi OA \& Blum RW (2008): "Predictors of early sexual initiation among a nationally representative sample of Nigerian adolescents". BioMed Central Public Health, 8: 136.

Fatusi, OA, \& Hindin, MJ (2010). Adolescents and youth in developing countries: Health and development issues in context. J Adolesc, 33(4), 499-508.

Federal Ministry of Health (FMoH) $(2010$, December). National HIV Sero Prevalence Sentinel

Lowndes, CM, Alarly, M; Bellau M; Bosu, WK; Kintin, DF; Nnorom, JA; Seck K; Victor-Ahuchogu, J \& Wilson, D (2008): "Western Africa HIV/AIDS epidemiology and response synthesis: Characterization of the HIV epidemic and response in West Africa: implications for prevention. Washington, DC, World Bank

Odu BK \& Akanle FF (2008): "Knowledge of HIV/ AIDS and sexual behaviour among the youths in South West Nigeria". Humanity \& Social Sciences Journal 3 (I): $8 \mathrm{I}-88$

Letamo, G (20II): "Does correct knowledge about HIV and AIDS lead to safer sexual behaviour? The case of young people in Bostwana". Journal of African Population Studies Vol 25, I (April 20 I I): 44-62

Omeonu PE \& Kollie ES (2010): "Knowledge and attitude of Babcock University students on risk behaviours of HIV/AIDS": Journal of Life and Physical Sciences 3(2): 135 - 142.

Onayade AA, Abiona TC, Ugbala C, Alozie G and Adetuyi O (2008): "Determinants of consistent condom use among adolescents and young adults attending a tertiary educational institution in lleIfe, Nigeria". Niger Postgrad Med Journal. 2008 Sep; I5(3): 185-19|

Oyediran, KA, Feyisetan, $\mathrm{Ol}$ and Akpan, T (20I I): "Predictors of Condom-use among Young Nevermarried Males in Nigeria". Journal Health Population Nutrition. 20I I June; 29(3): 273-285.

Santelli, J, Kaiser J, Hirsch L, Radosh A, Simkin L, \& Middlestadt S (2004): "Initiation of sexual intercourse among middle school adolescents: The influence of psychosocial factors". Journal of Adolescent Health; 34:200-208.

UNAIDS (2008): Report on the global AIDS epidemic, Geneva

Voeten, H; Egesah, OB \& Habbema JD (2004): "Sexual Behavior is more risky in rural than in urban areas among young women in Nyanza Province, Kenya". Journal of the American Sexually Transmitted Diseases: Volume 31 - Issue 8 - pp 48I-487 